\title{
Role of resveratrol and chrysin on inflammation and liver function in obese mice
}

DOI:10.36909/jer.ASSEEE.16071

Pipit Pitriani $^{1, *}$, Wang-Lok Lee ${ }^{2}$, Hee-Geun Park $^{3}$

${ }^{1}$ Faculty of Sport and Health Education, Universitas Pendidikan Indonesia, Bandung, Indonesia

${ }^{2}$ Department of Sports Science, College of Natural Science, Chungnam National University, Daejeon, Korea

${ }^{3}$ Korea Center for Sport Science in Jeonbuk, Jeonju, Korea

*Email: pipitpitriani@ upi.edu; Corresponding Author.

\begin{abstract}
The incidence of obesity has been spreading throughout the world. Many of the complications caused by obesity, such as inflammation and impaired liver function. This study aimed to determine the effect of supplementation resveratrol and chrysin on inflammation and liver function of obese mice fed a high-fat diet. 40 mice (C57BL/6) were randomly divided into four groups: 10 in the normal diet (NC), 10 control group on a highfat diet (HC), 10 in the high-fat diet with resveratrol (HRE), and 10 in the high-fat diet group with chrysin $(\mathrm{HCH})$. Resveratrol $25 \mathrm{mg}$ and 50mg of chrysin supplement per kg body weight were orally given with $0.1 \mathrm{ml}$ solution of Dimethyl Sulfoxide (DMSO) dissolved in for 15 weeks (4 times/week). The calorie intake of the group supplemented by resveratrol and chrysin significantly decreased. Group with high-fat diet, resveratrol, and chrysin increased body weight significantly compared to the normal diet group. The liver weight decreased in the resveratrol but not in the chrysin group. TNF $\alpha$ did not decrease in the resveratrol and chrysin group while IL1 $\beta$ significantly decreased. TLR 4 significantly decreased only in the chrysin group, while IL10 only increased in the resveratrol group. The collagen was
\end{abstract}


decreased by resveratrol and chrysin supplementation while fibronectin was not affected by resveratrol or chrysin. The inflammatory process in the liver of obese mice fed a high-fat diet can be reduced by supplementing resveratrol and chrysin.

Keywords: chrysin, inflammation, liver function, obese, resveratrol

\section{INTRODUCTION}

Excess body fat is a risk factor for obesity that leads to metabolic diseases and its incidence continues to increase throughout the world over the last 30 years (Hotamisligil et al., 2008). Enlarged adipose cells cause altered tissue function and augmented pro-inflammatory adipokines.(Romacho et al, 2014).

Nonalcoholic fatty liver disease (NAFLD) characterized by increased fat content spans a wide range of histologic surroundings (Braunersreuther et al., 2012). Hepatic lipid accumulation from the accelerated influx of free fatty acids (FFA) and de novo lipid synthesis can form fatty liver (Dietrich et al., 2014; Martín-Domínguez et al., 2013). Oxygen free radicals (OFR) generated by free fatty acids in mitochondria cause lipid peroxidation and induce the synthesis of pro-inflammatory cytokines due to Kupffer cells and hepatocytes, such as TNF- $\alpha$, TGF- $\beta 1$, FAS ligands and IL-8 (Martín-Domínguez et al., 2013). Liver injury and fibrosis are induced by TNF- in the process of NAFLD development. TNF- $\alpha$ regulates hepatocytes TGF- $\beta$ and $\alpha$-SMA and then TGF- $\beta$ persuades TIMP 1 , that promotes the development of liver fibrosis through induction of collagen $1 \alpha$ expression (Tomita et al., 2006; Wang et al. 2009). Collagen $1 \alpha$ and TIMP1 gene expression were bigger in the liver of C57BL/6 mice fed a high-fat and high-fructose water (HFF) diet (Kawanishi et al., 2012). Resveratrol is a polyhenol stilbenes which includes antioxidant compounds and can reduce hepatic oxidative stress in diabetic rodents and the NAFLD model as well as an antiinflammatory which acts on AMP-activated protein kinase and SIRT1 activator (Bujanda et 
al., 2008). Disruption of the Keap1/Nrf2 pathway is a potential mechanism to upregulate antioxidant enzymes as well as inhibit LPS reactivity caused by CD-14 in Kupffer cells (Kessoku et al., 2016; Li et al., 2014).

Chrysin (5,7-dihydroxyflavone) is a naturally occurring flavonoid with anti-inflammatory and antioxidant properties found in many plant extracts and dietary supplements, including honey and propolis (Mani et al., 2018). Chrysin as an anti-inflammatory can be used in various neurodegenerative diseases (Lee et al., 2017). Macrofiltration into adipose tissue and changes in M1/M2 status are the effects of chrysin administration on fat (Feng et al., 2014). Calcium release, nitric oxide (NO) production, various cytokines, and expression of CHOP and Fas mRNA in dsRNA [polyinosinic-polysitidylic acid] significantly inhibited their production (Lee et al., 2015). Chrysin induces the formation of brown adipose cells and increases fat metabolism, so it is considered as a supplement to prevent obesity (Choi et al., 2016).

Recently, functional nutrition diets are used to treat obesity induced metabolic complication. Up to now the effect of resveratrol and chrysin on the inflammation process and lipid metabolism have been established, however, the effects on liver function when obesity occurred still not many discovered. Therefore, the study aim was to explore the chrysin and resveratrol diet effects on inflammation and liver function of obese mice.

\section{MATERIAL AND METHOD}

In the animals and diet, 40 mice Male C57BL/6 age 4 weeks old were housed in cages (5 mice per cage) (Central Experimental Animal, Korea), at temperature $22 \pm 2^{\circ} \mathrm{C}$, with $60 \pm 5 \%$ humidity. Adaptation period 1 week then feed either a high fat diet consists of $60 \%$ calories from fat product of Orient Bio Inc., \#D12492 or a normal diet contain 18\% calories from fat, product of Orient Bio Inc., \#2018 ad libitum for 15 weeks.

The mice were split into four groups: control normal diet $(\mathrm{NC} ; \mathrm{n}=10)$, high fat diet control 
$(\mathrm{HC} ; \mathrm{n}=10)$, resveratrol supplement $(\mathrm{HRE} ; \mathrm{n}=10)$ and chrysin supplement $(\mathrm{HCH} ; \mathrm{n}=10)$ groups. Calculation of body weight and food intake are carried out every week In the resveratrol and chrysin supplements, resveratrol and chrysin was purchased from Sigma Aldrich Inc. $25 \mathrm{mg} / \mathrm{kg}$ body weight of Resveratrol and $50 \mathrm{mg} / \mathrm{kg}$ body weight of chrysin dissolved in $0.1 \mathrm{ml}$ Dimethyl Sulfoxide (DMSO) solution were administered orally using a single-use $1 \mathrm{ml}$ syringe at a dose of $0.1 \mathrm{~mL}$ per mice 4 times a week for 15 weeks. Mice were sacrificed under anesthesia using a mixture of ketamine $(80 \mathrm{mg} / \mathrm{kg})$ and xylazine $(10 \mathrm{mg} / \mathrm{kg})$ after fasting for 12 hours. Liver tissue was taken, weighed and frozen in liquid nitrogen and then stored at $-80^{\circ} \mathrm{C}$.

In the real-time quantitative PCR, $50 \mathrm{~g}$ liver tissue homogenate with $1 \mathrm{ml}$ Trizol (Ambion, Carlsbad, CA, USA). The total RNA was calculated by ultraviolet spectrophotometry measuring absorbance at $260 \mathrm{~nm}$ and $280 \mathrm{~nm}$. Maxi RT PreMix kit (iNtRON, Korea) was used for cDNA synthesis. Polymerase Chain Reaction (PCR) were performed with the CFX 96 touch real-time PCR detection system using Smobio ExcelTaq 2X Q-PCR Master Mixed. The housekeeping gene Glyceraldehyde-3-phosphate dehydrogenase (GAPDH) mRNA was used, and all data characterized comparative to its expression. The primer sequences for each gene are shown in Table 1. Statistical data analysis using one-way ANOVA through post hoc LSD test was performed with SPSS V22.0. Statistical significance was defined as $=0.05$.

Table 1. The PCR primer sequences for each gene

\begin{tabular}{|l|l|l|}
\hline Gene & Forward & Reverse \\
\hline Collagen $1 \alpha$ & GGAATCCGGGGTTTACCTGG & AGGCGACCCTCTGATACCTT \\
\hline Fibronectin & GAGCCGGACAACTTCTGGTC & ATTTGCTGAGCCTGCCTCTT \\
\hline TNF $\alpha$ & TGAGAAGTTCCCAAATGGC & GCTACAGGCTTGTCACTC \\
\hline IL-1 $\beta$ & TCACAAGCAGAGCACAAG & GAAACAGTCCAGCCCATAC \\
\hline IL-10 & ATACTGCTAACCGACTCCTT & CAAATGCTCCTTGATTTCTGG \\
\hline TLR4 & GATCATGGCACTGTTCTTCT & AGGAATGTCATCAGGGACTT \\
\hline GAPDH & GGAGAGTGTTTCCTCGTCC & AATGAAGGGGTCGTTGATGG \\
\hline
\end{tabular}




\section{RESULTS}

Characteristic changes are

(i) Calorie Intake. The calorie intake was shown in Figure 1. The calorie intake in the high-fat diet group increased significantly while in the resveratrol and chrysin groups decreased significantly $(\mathrm{p}=0.00)$.

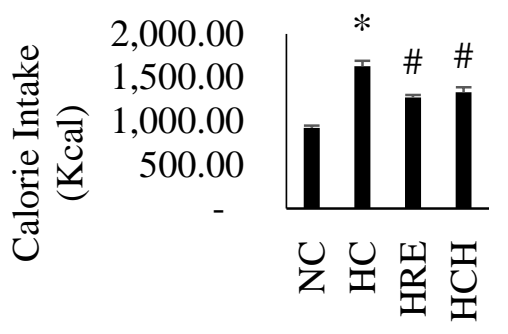

FIGURE 1 The effects of resveratrol and chrysin on calorie intake of obese mice. Values represent means \pm SEM. * compared to $\mathrm{NC}(\mathrm{p}<0.05)$, \#compared to $\mathrm{HC}(\mathrm{p}<0.05) . \mathrm{NC}=$ Normal Diet Control, HC = High Fat Diet, HRE = High Fat with Resveratrol, and HCH = High Fat with Chrysin

(ii) Body weight. The body weight was shown in Figure 2. The body weight increased in all groups by feeding a high fat diet for 15 weeks significantly.

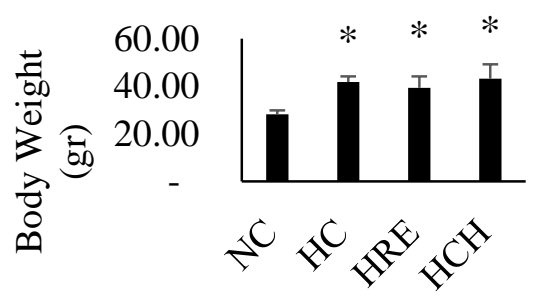

FIGURE 2 The effect of resveratrol and chrysin on body weight of obese mice. *Compared to $\mathrm{NC}(\mathrm{p}<0.05)$ 
(iii) Liver weight. The liver weight was shown in Figure 3. The liver weight of the high fat diet control group was increased significantly $(\mathrm{p}=0.00)$. The liver weight of a high fat diet with a resveratrol group was decreased significantly $(\mathrm{p}<0.01)$. However, the liver weight of chrysin group was not decrease significantly.

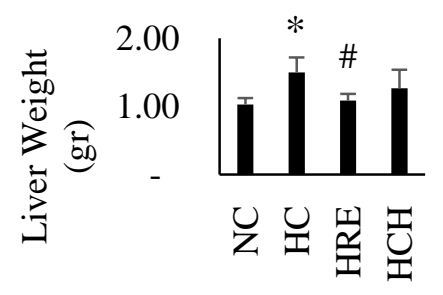

FIGURE 3 The effects of resveratrol and chrysin on the liver weight of obese mice.

Inflammatory cytokine gene expression is explained in the following:

(i) TNF- $\alpha$ gene expression. The result of TNF $\alpha$ gene expression was shown in Figure 4. The high fat diet control significantly increased the TNF $\alpha$ gene expression $(p<0.01)$. However, the resveratrol group, and high fat diet with chrysin groups were slightly reduced compared to high diet control but not significant.

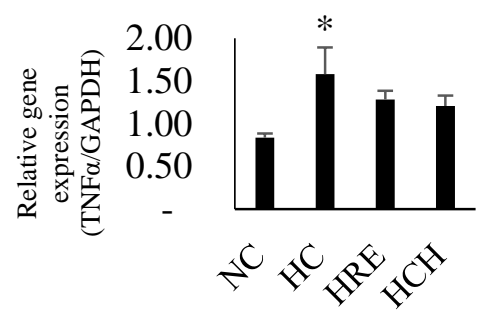

FIGURE 4 Effects of resveratrol and chrysin on gene expression of TNF $\alpha$ in obese mice with high fat diet. Value represent means \pm SEM.

(ii) IL-1 $\beta$ gene expression. The result of IL-1 $\beta$ gene expression was shown in Figure 5 . The IL-1 $\beta$ gene expression significantly increased in high fat diet control group $(p=0.00)$. The 
gene expression of IL- $1 \beta$ decreased in resveratrol and chrysin groups significantly $(p=0.00)$. The result shows that resveratrol, and chrysin supplement can eliminate the IL-1 $\beta$ gene expression in obese mice constrained high fat diet.

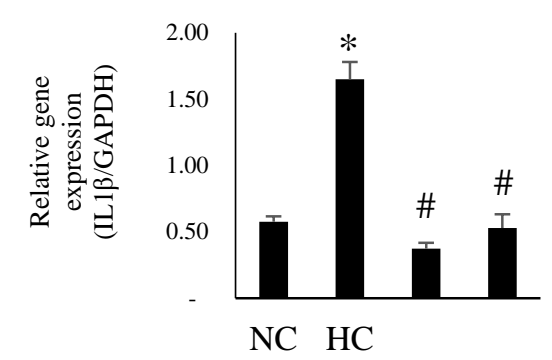

FIGURE 5 Effects of resveratrol and chrysin on gene expression of IL1 $\beta$ in obese mice. Value represent means \pm SEM.

(iii) TLR4 gene expression. The result of TLR4 gene expression was shown in Figure 6. The TLR4 gene expression did not increase in the high fat diet control group $(\mathrm{p}=0.794)$. The TLR4 decreased in chrysin group significantly $(\mathrm{p}<0.05)$, however, resveratrol group did not reduce the gene expression of TLR4.

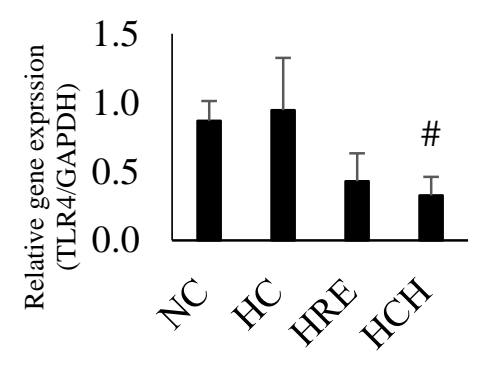

FIGURE 6 Effects of chrysin and resveratrol on TLR4 of obese mice. Values represent mean \pm SEM.

(iv) IL-10 gene expression. The IL-10 gene expression was shown in Figure 7. The IL-10 gene expression reduced in the control group with high fat diet $(\mathrm{p}=0.00)$. Resveratrol significantly increased the gene expression of IL-10 $(\mathrm{p}<0.05)$, however, chrysin did not 
significantly increase the IL-10 gene expression. The resveratrol supplementation increased the IL-10 gene expression more compared to chrysin in high fat diet induced obese mice.

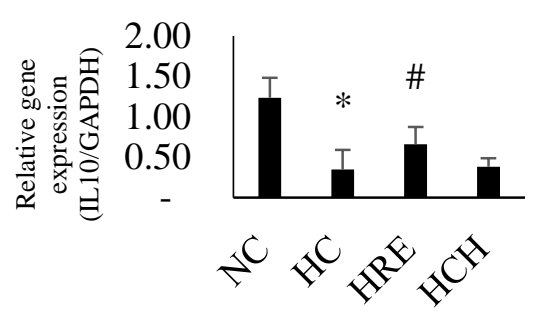

FIGURE 7 The effects of resveratrol and chrysin on the IL-10 gene expression of $t$ obese mice. Value represent means \pm SEM.

(v) Collagen $1 \alpha$ gene expression. The Collagen $1 \alpha$ gene expression was shown in Figure 8 . The collagen $1 \alpha$ gene expression increased in control group with high fat diet significantly $(p<0.05)$. The gene expression of collagen $1 \alpha$ decreased in the resveratrol and chrysin group significantly.

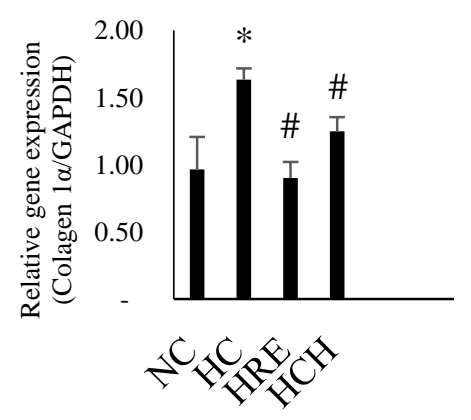

FIGURE 8 The effects of chrysin and resveratrol on colagen $1 \alpha$ in high fat diet induced obese mice. Value represent means \pm SEM.

(vi) Fibronectin gene expression. The fibronectin gene expression was shown in Figure 9. The fibronectin gene expression increased in the control group with high fat diet significantly 
$(\mathrm{p}<0.01)$. The expression of the fibronectin gene in the resveratrol and chrysin groups was only slightly reduced and did not show significant changes.

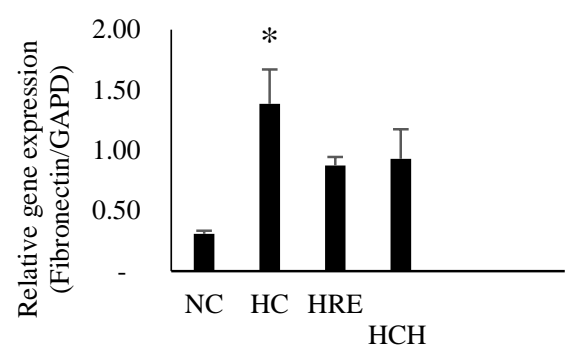

FIGURE 9 The effects of resveratrol and chrysin on fibronectin in obese mice. Values represent mean \pm SEM.

\section{DISCUSSION}

A diet high in fat 32-60\% total calories can induce obesity in rodents (Chang et al., 2015; Cintra et al., 2008). In the current study, we used $18 \%$ of calories from fat for normal group and $60 \%$ of calories from fat for high fat diet group for 15 weeks. The calorie intake of resveratrol and chrysin supplement group significantly decreased (Figure 1).

We found that the body weight of C57BL/6J mice increased in the control group with high fat diet significantly (Figure 2), however, the body weight was not reduced significantly by resveratrol and chrysin supplement groups. The resveratrol $10 \mathrm{mg} / \mathrm{kg}$ had no significant effect on body weight in previous study (Jun et al., 2014). Resveratrol given at different doses of 2, 10 or $20 \mathrm{mg} / \mathrm{kg} / \mathrm{day}$ gave ineffective results in weight loss in Zucker rats and mice (Rivera et al., 2009., Kessoku et al., 2016). This finding contradicts other findings that weight gain can reduce in rats and mice fed a high-fat diet with resveratrol (Cho et al., 2012). Resveratrol $25 \mathrm{mg} / \mathrm{kg}$ of mice body weight were given orally but the body weight was not reduced significantly. 
Chrysin supplementation did not reduce body weight, which is in line with previous studies showing that chrysin can reduce inflammation caused by a high-fat diet and regulate M1/M2 macrophage status in mice without altering body weight (Feng et al., 2014).

The recent result show that resveratrol supplement decreased more liver weight compared to chrysin supplement group in obese mice with high fat diet. This result was similar with previous study that reported resveratrol can reduce liver weight in high fat diet mice (Kessoku et al., 2016).

The recent study found that resveratrol and chrysin supplement did not significantly reduce the TNF- $\alpha$ in high fat diet group. TNF- as a precursor for the development of NASH by inducing liver injury and fibrosis (Zhang et al., 2010). Several studies reported that IL-1 $\beta$ and TNF- forced cell apoptosis in NASH model mice through activation of pro-apoptotic proteins. The recent study found that resveratrol and chrysin supplement decrease the gene expression of IL-1 $\beta$. Other study showed that chrysin can significantly reduce the releasing of proinflammatory cytokine IL-1 $\beta$ in serum from obese mice (Feng et al., 2014). The recent study showed that chrysin supplementation reduce more the gene expression of TLR4 compared to resveratrol supplement, in high fat diet induced obese mice.

IL-10 as anti-inflammatory cytokine inhibits inflammation in numerous organs including T cells, monocytes, macrophages and protects liver steatosis (Den Boer et al., 2006, Braunersreuther et al., 2012). Impaired insulin signaling, steatosis and increased expression of F4/80, TNF- $\alpha$, IL-1 $\beta$ as well as IL-6, may occur with inhibition of either anti-IL-10 antibodies or antisense IL-10 oligonucleotides (Cintra et al., 2008). Our study found that resveratrol supplement reduced more the IL-10 gene expression compared to chrysin supplement. C57BL/6 mice high-fat diet (HFD) with resveratrol showed a dose-dependent increase in IL-10 (Wang et al., 2013). 
Liver stealic cells (HSCs) promote fibrosis by increasing the production matrix per cell consisting mainly of collagens IV as well as collagen VI, which is increasingly supported by collagens I, collagens III as well as cellular fibronectin (Lee et al., 2011).

Liver fibrosis occurs with changes in the quantity and composition of ECM resulting from increased and decreased synthesis (Duarte et al., 2015, Pincu et al., 2015). Resveratrol supplementation can reduce Collagen $1 \alpha$ more than chrysin supplementation in obese mice force by high fat diet. Another finding showed that resveratrol blocked fibrosis process and inflammation dramatically but not steatosis in a NASH model of mice (Kessoku et al., 2016). Fibronectin formed by hepatic stellate cells (HSCs), is glycoprotein with many function and ECM found in the cytoplasm as well as in cell membrane. Fibronectin occurs prior to collagen deposition (Liu et al., 2016). Fibronectin is also present in the fibrotic matrix which is required for collagen matrix assembly in vitro. It also regulates the amount of growth factors and unlocks them from the matrix. The resveratrol and chrysin supplementation can decrease the fibronectin gene expression.

\section{CONCLUSION}

Resveratrol and chrysin as polyphenol supplementation have many effects in inflammation and liver disorder. In our study, the process of inflammation in mice liver that fed by high fat diet induced obese could decrease by supplementation of resveratrol and chrysin. The liver function can change mostly by resveratrol supplementation.

\section{ACKNOWLEDGMENTS}

This research was funded by the National Research Foundation of Korea Grant (NRF2013S1A5B5A07048323). 


\section{REFERENCES}

Braunersreuther, V., Viviani, G. L., Mach, F., \& Montecucco, F. 2012. Role of cytokines and chemokines in non-alcoholic fatty liver disease. World Journal of Gastroenterology, $18(8), 727-735$.

Bujanda, L., Hijona, E., Larzabal, M., Beraza, M., Aldazabal, P., García-Urkia, N., ... Arenas, J. I. 2008. Resveratrol inhibits nonalcoholic fatty liver disease in rats. BMC Gastroenterology, 8(40), 1-8.

Chang, C.-C., Lin, K.-Y., Peng, K.-Y., Day, Y.-J., \& Hung, L.-M. 2015. Resveratrol exerts anti-obesity effects in high-fat diet obese mice and displays differential dosage effects on cytotoxicity, differentiation, and lipolysis in 3T3-L1 cells. Endocrine Journal, 63(2), 169-178.

Cho, S.-J., Jung, U. J., \& Choi, M.-S. 2012. Differential effects of low-dose resveratrol on adiposity and hepatic steatosis in diet-induced obese mice. British Journal of Nutrition, 108(12), 2166-2175.

Choi, J. H., \& Yun, J. W. 2016. Chrysin induces brown fat-like phenotype and enhances lipid metabolism in 3T3-L1 adipocytes. Nutrition, 32(9), 1002-1010.

Cintra, D. E., Pauli, J. R., Araújo, E. P., Moraes, J. C., de Souza, C. T., Milanski, M., \&Velloso, L. A. 2008. Interleukin-10 is a protective factor against diet-induced insulin resistance in liver. Journal of Hepatology, 48(4), 628-637.

Dietrich, P., \& Hellerbrand, C. 2014. Non-alcoholic fatty liver disease, obesity and the metabolic syndrome. Best Practice \& Research Clinical Gastroenterology, 28(4), 637-653.

Den Boer, M. A., Voshol, P. J., Schröder-van der Elst, J. P., Korsheninnikova, E., Ouwens, D. M., Kuipers, F., \& Romijn, J. A. 2006. Endogenous interleukin-10 protects 
against hepatic steatosis but does not improve insulin sensitivity during high-fat feeding in mice. Endocrinology, 147(10), 4553-4558.

Duarte, S., Baber, J., Fujii, T., \& Coito, A. J. 2015. Matrix metalloproteinases in liver injury, repair and fibrosis. Matrix Biology, 44-46, 147-156.

Feng, X., Qin, H., Shi, Q., Zhang, Y., Zhou, F., Wu, H., ... Shen, P. 2014. Chrysin attenuates inflammation by regulating M1/M2 status via activating PPAR?? Biochemical Pharmacology, 89(4), 503-514.

Hotamisligil, G. S., \& Erbay, E. 2008. Nutrient sensing and inflammation in metabolic diseases. Nature Reviews Immunology, 8(12), 923-934.

Jun, J. K., Lee, W. L., Park, H. G., Lee, S. K., Jeong, S. H., \& Lee, Y. R. 2014. Moderate intensity exercise inhibits macrophage infiltration and attenuates adipocyte inflammation in ovariectomized rats. 18(1), 119-127.

Kawanishi, N., Yano, H., Mizokami, T., Takahashi, M., Oyanagi, E., \& Suzuki, K. 2012. Exercise training attenuates hepatic inflammation, fibrosis and macrophage infiltration during diet induced-obesity in mice. Brain, Behavior, and Immunity, 26(6), 931-941.

Kessoku, T., Imajo, K., Honda, Y., Kato, T., Ogawa, Y., Tomeno, W., \&Nakajima, A. 2016. Resveratrol ameliorates fibrosis and inflammation in a mouse model of nonalcoholic

Lee, B. K., Lee, W. J., \& Jung, Y. S. 2017. Chrysin attenuates VCAM-1 expression and monocyte adhesion in lipopolysaccharide-stimulated brain endothelial cells by preventing NFผB signaling. International Journal of Molecular Sciences, 18(7), 1-12. 
Lee, J. Y., \& Park, W. 2015. Anti-inflammatory effect of chrysin on RAW 264.7 mouse macrophages induced with polyinosinic-polycytidylic acid. Biotechnology and Bioprocess Engineering, 20(6), 1026-1034.

Lee, U. E., \& Friedman, S. L. 2011. Mechanisms of hepatic fibrogenesis. Best Practice and Research: Clinical Gastroenterology, 25(2), 195-206.

Li, L., Hai, J., Li, Z., Zhang, Y., Peng, H., Li, K., \& Weng, X. 2014. Resveratrol modulates autophagy and NF-??B activity in a murine model for treating non-alcoholic fatty liver disease. Food and Chemical Toxicology, 63, 166-173.

Liu, X. Y., Liu, R. X., Hou, F., Cui, L. J., Li, C. Y., Chi, C., \& Yin, C. H. 2016. Fibronectin expression is critical for liver fibrogenesis in vivo and in vitro. Molecular Medicine Reports, 14(4), 3669-3675.

Mani, R., \& Natesan, V. 2018. Chrysin: Sources, beneficial pharmacological activities, and molecular mechanism of action. Phytochemistry, 145, 187-196.

\section{Martín-Domínguez, V., González-Casas, R., Mendoza-Jiménez-Ridruejo, J., García-}

Pincu, Y., Linden, M. A., Zou, K., Baynard, T., \& Boppart, M. D. 2015. The effects of high fat diet and moderate exercise on TGF $\beta 1$ and collagen deposition in mouse skeletal muscle. Cytokine, 73(1), 23-29.

Rivera, L., Morón, R., Zarzuelo, A., \& Galisteo, M. 2009. Long-term resveratrol administration reduces metabolic disturbances and lowers blood pressure in obese Zucker rats. Biochemical pharmacology, 77(6), 1053-1063.

Romacho, T., Elsen, M., Röhrborn, D., \& Eckel, J. 2014. Adipose tissue and its role in organ crosstalk. Acta physiologica, 210(4), 733-753. 
Tomita, K., Tamiya, G., Ando, S., Ohsumi, K., Chiyo, T., Mizutani, A., \& Hibi, T. 2006. Tumour necrosis factor $\alpha$ signalling through activation of Kupffer cells plays an essential role in liver fibrosis of non-alcoholic steatohepatitis in mice. Gut, 55(3), 415-424.

Wang, J., Leclercq, I., Brymora, J.M., Xu, N., Ramezani-Moghadam, M., London, R.M., Brigstock, D. and George, J., 2009. Kupffer cells mediate leptin-induced liver fibrosis. Gastroenterology, 137(2), 713-723.

Wang, B., Sun, J., Li, X., Zhou, Q., Bai, J., Shi, Y., \& Le, G. 2013. Resveratrol prevents suppression of regulatory T-cell production, oxidative stress, and inflammation of mice prone or resistant to high-fat diet-induced obesity. Nutrition Research, 33(11), 971-981. 